\title{
$\mathrm{R}$ \\ Revista d \\ de \\ Saúde

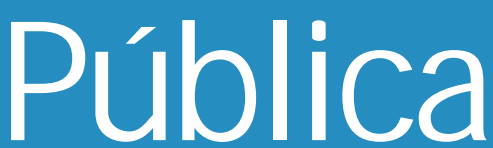

$\begin{array}{lllllll}\mathbf{J} & \mathbf{U} & \mathbf{R} & \mathbf{N} & \mathbf{A} & \mathbf{L}\end{array}$

$0 F$

$\mathbf{P} \mathbf{U}$

$B \quad L \quad$ I C

H E A L T H

\section{H ospitalização por pneumonia: influência de fatores socioeconômicos e gestacionais em uma coorte de crianças no Sul do Brasil}

\section{Hospitalizations due to pneumonia: the influence of socioeconomic and pregnancy factors in a cohort of children in Southern Brazil}

Juraci A. César, Cesar G. Victora, Iná S. Santos, Fernando C. Barros, Elaine P. Albernaz, Luciani M. O liveira, José A. Flores, Bernardo L. Horta, Elizabete Weiderpass e Ricardo Halpern

Departamento M aterno-Infantil da U niversidade do Rio Grande. Rio Grande, RS - Brasil (J.A.C., B.L.H.); D epartamento de Medicina Social da Universidade Federal de Pelotas. Pelotas, RS - Brasil (C.G.V., I.S.S., F.C.B., E.W.); Fundação de Apoio U niversitário. Pelotas, RS - Brasil (E.P.A., L.M .O .); Hospital Pediátrico Santo Antonio. Porto Alegre, RS - Brasil (J.A.F.); D epartamento Materno-Infantil da U niversidade Federal de Pelotas. Pelotas, RS Brasil (R.H.) 


\title{
H ospitalização por pneumonia: influência de fatores socioeconômicos e gestacionais em uma coorte de crianças no Sul do Brasil*
}

\section{Hospitalizations due to pneumonia: the influence of socioeconomic and pregnancy factors in a cohort of children in Southern Brazil}

\author{
Juraci A. César, Cesar G. Victora, Iná S. Santos, Fernando C. Barros, Elaine P. Albernaz, \\ Luciani M. O liveira, José A. Flores, Bernardo L. H orta, Elizabete W eiderpass e Ricardo \\ Halpern \\ Departamento Materno-Infantil da U niversidade do Rio Grande. Rio Grande, RS - Brasil (J.A.C., \\ B.L.H.); D epartamento de M edicina Social da U niversidade Federal de Pelotas. Pelotas, RS - Brasil \\ (C.G.V., I.S.S., F.C.B., E.W.); Fundação de Apoio U niversitário. Pelotas, RS - Brasil (E.P.A., L.M .O .); \\ Hospital Pediátrico Santo Antonio. Porto Alegre, RS - Brasil (J.A.F.); Departamento Materno-Infantil \\ da U niversidade Federal de Pelotas. Pelotas, RS - Brasil (R.H.)
}

\begin{abstract}
Resumo
Objetivo

Investigar a influência de fatores socioeconômicos e gestacionais sobre a hospitalização por pneumonia no período pós-neonatal.

Material e Método

Longitudinal.

Crianças com idade entre 28 e 364 dias, nascidas na cidade de Pelotas, RS (Brasil), em 1993. A definição de caso foi a permanência em ambiente hospitalar por um período igual ou superior a 24 horas em conseqüência de pneumonia. Foi aplicado delineamento longitudinal.

Resultados Dentre as 5.304 crianças da coorte, 152 (2,9\%) foram hospitalizadas por pneumonia no período. O valor preditivo positivo do diagnóstico clínico comparado com o radiológico alcançou 76\%. A análise através de regressão logística mostrou que a classe social e a escolaridade materna estiveram forte e inversamente associadas à admissão hospitalar. Filhos de mães adolescentes tiveram risco duplicado à internação; paridade igual ou superior a três representou risco 2,8 vezes maior em relação às mães primíparas; ganho de peso inferior a $10 \mathrm{~kg}$ durante a gestação implicou risco cerca de $40 \%$ maior à hospitalização.

Conclusões A classe social e a escolaridade materna foram os principais determinantes da hospitalização. Idade e paridade materna e o ganho de peso durante a gestação foram também fatores de risco importantes.
\end{abstract}

Pneumonia, epidemiologia. Hospitalização. Fatores de risco. Fatores socioeconômicos.

\footnotetext{
* Projeto financiado pela Comunidade Econômica Européia, Organização Mundial da Saúde, Fundação de Amparo à Pesquisa do Rio Grande do Sul de Aperfeiçoamento de Pessoal de Nível Superior.

Correspondência para/Correspondence to: Juraci A. César - Departamento Materno-Infantil da Universidade do Rio Grande. R. Eng. Alfredo Huch, 475 - 96201-900 Rio Grande, RS - Brasil. E-mail: jura @ vetorial net. com.br

Recebido em 18.12.1995. Reapresentado em 23.5.1995. Aprovado em 12.8.1996.
} 


\begin{abstract}
Objective To investigate the influence of socioeconomic and gestational factors on admission due to pneumonia in the post-neonatal period.

Methodology Cohort.

Children born in the city Pelotas, Brazil, in 1993. Cases were children admitted to hospital for 24 hours or more, between the ages of 28 and 364 days, with a diagnosis of pneumonia.

Results Of the 5,304 children in the cohort, 152(2.9\%) were hospitalized with pneumonia. The positive preditive value of the clinical diagnosis compared to the radiological assessment was $76 \%$. Analysis by conditional logistic regression showed that social class and maternal schooling were strongly inversely associated with pneumonia. Children of adolescent mothers were twice as likely to be admitted. The relative risk for children whose mothers were of parity three or greater was 2.8 relative to primiparae. Maternal weight gain during pregnancy of less than $10 \mathrm{~kg}$ was associated with a $40 \%$ increase in risk.
\end{abstract}

Conclusion Socioeconomic factors were important determinants of pneumonia admissions. Maternal age, parity and weight gain were also significant risk factors.

Pneumonia, epidemiology. Hospitalization. Risk factors. Socioeconomic factors.

\section{INTRO DUÇÃO}

As infecções respiratórias agudas são responsáveis por um terço das mortes e metade das hospitalizações e consultas médicas entre menores de cinco anos nos países em desenvolvimento ${ }^{5,18,23}$. A pneumonia, sua mais grave conseqüência, mata cerca de 800 crianças a cada hora, com 53\% desses óbitos ocorrendo no período pós-neonatal ${ }^{5,23}$.

Dentre os diversos fatores de risco identificados estão os de baixo nível socioeconômico, desnutrição, baixo peso ao nascer, ausência de amamentação, aglomeração familiar, poluição no ambiente e no domicílio $^{18,19,23,26,32}$. Mais recentemente, foram também implicados ${ }^{11,30}$ frequiência a creches e episódios prévios de chiado no peito.

A maioria desses fatores tem sido estudada em relação à incidência e mortalidade por pneumonia ${ }^{11,18,23,27,30}$. No entanto, poucos estudos encontramse disponíveis sobre a ocorrência de hospitalizações, um evento de custo elevado, bastante freqüente e quase sempre precedendo a morte ${ }^{18,23,28}$. Isto dificulta que medidas específicas sejam adotadas visando a reduzir a gravidade dos casos e, conseqüentemente, diminuir a contribuição dessa doença no coeficiente de mortalidade infantil.

O presente estudo visa a conhecer a influência de fatores socioeconômicos e gestacionais sobre a hospitalização por pneumonia, no período pós-neonatal, em uma coorte de base populacional no Sul do Brasil.

\section{METO DOLO GIA}

Com cerca de 300 mil habitantes, Pelotas é a maior cidade do extremo sul do Brasil ${ }^{12}$; apresenta clima temperado quente com temperatura anual média de $17,9^{\circ} \mathrm{C}$ e umidade relativa do ar média de $80,3 \%{ }^{10}$. Tem na indústria alimentícia e no comércio a base da sua economia; o produto interno bruto (PIB) per capita anual é de aproximadamente U\$ $2,700^{22}$. Noventa e dois por cento dos domicílios dispõem de água tratada e $44,3 \%$ de rede de esgotos ${ }^{12}$. A prestação de serviços em saúde, na quase sua totalidade, é feita de forma gratuita através de 43 unidades sanitárias básicas, cinco ambulatórios e cinco hospitais ${ }^{6}$. Apesar de toda essa estrutura, e de dispor um médico para cada 260 habitantes, cerca de $5 \%$ das mães não fazem pré-natal, $18,4 \%$ das crianças apresentam o esquema vacinal básico incompleto ao final do primeiro ano de idade e 21 delas morrem antes de completar o primeiro ano de vida ${ }^{31}$. Mais de $99 \%$ dos nascimentos da zona urbana ocorrem em hospital ${ }^{31}$.

A coorte da qual este estudo faz parte compreende os 5.304 nascidos vivos ocorridos na cidade de Pelotas, RS, no ano de 1993. As mães dessas crianças, quando ainda no hospital, eram entrevistadas por uma equipe do estudo perinatal quanto a aspectos demográficos, socioeconômicos, reprodutivos, ambientais e de utilização de serviços de saúde $^{31}$. Crianças portadoras de malformações congênitas (4), fibrose cística (1) e gemelares (2) foram excluídas.

O cálculo de tamanho da amostra foi baseado em um erro beta de 0,1 , erro alfa de 0,05 , exposição variando entre $20 \%$ e $50 \%$ e razão de incidência cumulativa de $2,0^{25}$. Acrescidos de $30 \%$ para controle de fatores de confusão e $10 \%$ para perdas, a pesquisa deveria cobrir pelo menos 4.547 crianças ${ }^{7}$. 
Cinco entrevistadores visitaram diariamente os setores de pronto socorro, enfermarias, berçário, unidade de tratamento intensivo e quartos particulares de todos os hospitais da cidade, de $1^{\circ}$ de janeiro de 1993 a 31 de dezembro de 1994. A cada três meses, os entrevistadores faziam rodízio para que atuassem em todos os hospitais; em caso de prontuário incompleto, o médico responsável era entrevistado.

Durante o período de internação questionava-se a mãe sobre os primeiros sinais e sintomas apresentados pela criança, tratamentos iniciais e busca de auxílio médico. Através da revisão de prontuário, coletaram-se informações sobre o período e o motivo da hospitalização, dados da anamnese, do exame físico, outros exames complementares, tratamentos recebidos e diagnóstico de alta. Cerca de $7 \%$ das revisões de prontuários e entrevistas com as mães foram repetidas pelo coordenador do estudo (JAC).

Foram incluídos como casos todas as crianças com idade entre 28 e 364 dias completos, nascidas na zona urbana de Pelotas, em 1993, que haviam permanecido em ambiente hospitalar por um período igual ou superior a 24 horas em decorrência de pneumonia.

Foi considerada como tendo pneumonia a criança que apresentasse tosse, disfunção respiratória (dificuldade respiratória, taquipnéia, retração inter e subcostal, etc), estertores crepitantes e, sempre que possível, alterações laboratoriais (gasometria e contagem de leucócitos) e radiológicas ${ }^{13,14,23,24}$. O julgamento destes itens era feito por, pelo menos, dois árbitros pediatras (EPA, LMO) utilizando a Classificação Internacional das Doenças - 9ª Revisão ${ }^{17}$. Em caso de discordância, um terceiro árbitro $(\mathrm{RH})$ era requisitado.

Para uma subamostra de crianças cujo exame radiológico de tórax foi obtido pela equipe da pesquisa, a presença de infiltrado intersticial, segundo critério da OMS, avaliado por um radiologista pediátrico (JAF), definia o diagnóstico de pneumonia ${ }^{24}$.

Embora quase todos os fatores de risco coletados sejam auto-explicativos, alguns necessitam de esclarecimentos complementares:

Classe social: utilizou-se a classificaçao de Bronfam e col. ${ }^{3}$, baseada na renda familiar, escolaridade dos pais, ocupação do chefe da família (pessoa de maior renda) e inserção no mercado de trabalho. As seguintes classes (ou frações de classe) foram constituídas: burguesia, nova pequena burguesia, pequena burguesia tradicional, proletariado não típico, proletariado típico e subproletariado.

Renda familiar: valor recebido por todos os moradores do domicílio no mês anterior à entrevista, dividido pelo valor do salário-mínimo da época.

Escolaridade: última série completada com aprovação.

Ganho de peso durante a gestação: diferença entre o peso na ocasião do parto e o peso pré-gestacional referido pela mãe.

Retardo de crescimento intra-uterino: crianças com peso de nascimento inferior ao percentil 10 em relação ao sexo e idade gestacional segundo a curva de Willians e cols. ${ }^{33}$.

Nascimento pré-termo: crianças com idade inferior a 37 semanas avaliadas a partir de critérios somáticos e neurológicos, segundo o método proposto por Dubowitz e col. ${ }^{8}$

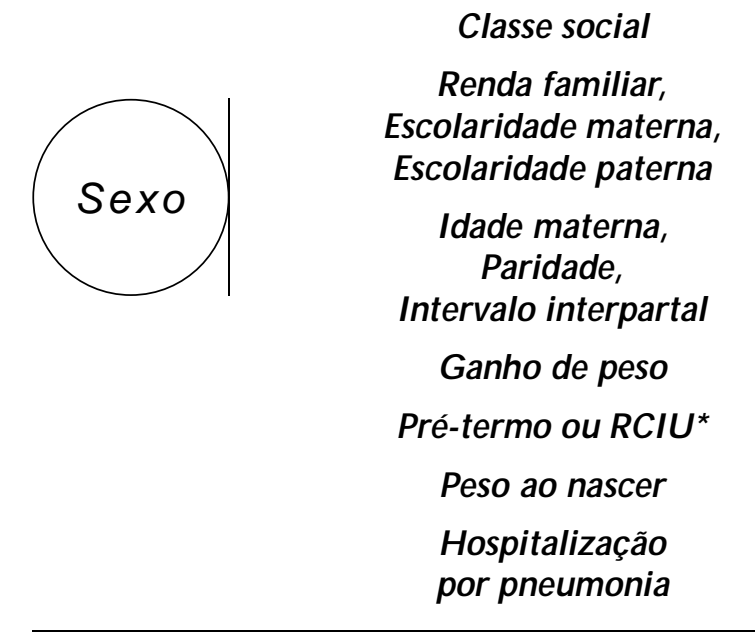

Figura - Modelo hierárquico para hospitalização por pneumonia

A análise inicial buscou associações entre os fatores de risco e a hospitalização por pneumonia, através do cálculo da razão de "odds" (e seu intervalo de confiança de $95 \%$ ) e do teste do qui-quadrado para tabelas de contingência. A análise multivariada, realizada através de regressão logística não condicional, obedeceu ao modelo hierárquico proposto na Figura. Assumiu-se que algumas variáveis independentes atuavam sobreterminando as demais. Assim, a classe social, situada no primeiro nível hierárquico, poderia influenciar todas as outras. O segundo nível foi constituído por renda e escolaridade dos pais, enquanto que o terceiro incluiu idade materna, paridade e intervalo interpartal. O nível seguinte incluiu ganho de peso durante a gestação e o último, retardo de crescimento intrauterino e nascimento pré-termo. A significância estatística da inclusão de cada variável foi aferida através do teste de razões de verossimilhança ${ }^{9}$. O modelo final incluiu todas as variáveis que, ao entrarem na equação - isto é, já ajustadas para as variáveis dos níveis superiores e do mesmo nível - apresentavam um valor $\mathrm{p}<0,10$. Cada variável ordinal (por exemplo, renda familiar em grupos) foi também testada para tendência linear; quando essa era significativa e não havia evidência de desvio da linearidade, a variável foi incluída como um componente linear.

Os valores desconhecidos, quando inferiores a 5\%, foram anexados à categoria mais freqüente; nos demais casos constituíam uma nova categoria, o que aconteceu somente com classe social, onde não se obteve informações em $7 \%$ das entrevistas.

\section{RESULTADOS}

As entrevistas hospitalares identificaram 6.410 nascimentos no ano de 1993, em Pelotas. Desses, $1.090(17,0 \%)$ eram filhos de mães residentes na zona rural ou provenientes de outras cidades e 16 $(0,3 \%)$ foram perdidos. Das 5.304 crianças estudadas, $152(2,9 \%)$ foram hospitalizadas por pneumo- 
nia no período pós-neonatal, totalizando 187 internações. O prontuário de quatro crianças $(2,6 \%)$ com pneumonia foram extraviados e não entraram no estudo ${ }^{31}$.

Dentre as crianças estudadas, $47(0,9 \%)$ tinham entre 28 e 89 dias na primeira hospitalização, 62 $(1,2 \%)$ entre 90 e 179 dias e $43(0,8 \%)$ entre 180 e 364 dias de idade. Houve um ligeiro predomínio de crianças do sexo masculino $(3,1 \%$ a $2,6 \%)$.

Cento e trinta e seis crianças (89\%) haviam sido submetidas a radiografia de tórax, das quais $70(46 \%)$ foram obtidas pela equipe da pesquisa. Dessas, somente $55(78 \%)$ apresentavam condições adequadas ao exame pelo radiologista segundo critérios da Organização Mundial da Saúde ${ }^{24}$. O valor preditivo positivo, isto é, a proporção de casos onde o diagnóstico clínico foi confirmado pelo radiológico atingiu $76 \%$ (42/55 x100). Dez outros exames radiográficos de crianças que, segundo avaliação dos árbitros clínicos, não apresentavam pneumonia, foram também submetidas ao radiologista juntamente com as demais. Para todas essas, o diagnóstico radiológico confirmou ausência de pneumonia.

$\mathrm{Na}$ análise bivariada, o risco de hospitalização por pneumonia esteve inversamente associado à classe social, renda familiar e escolaridade dos pais (Tabela 1); crianças pertencentes à pequena burguesia tradicional e subproletariado apresentaram risco 1,5 e 3,5 vezes maior, respectivamente, de serem hospitalizadas em relação à categoria-base (proletariado não típico). A razão de "odds" para crianças com renda familiar mensal $\leq$ a 1 salário-mínimo (SM) foi cerca de três vezes maior, enquanto que o grupo intermediário (1,1 a 3,0 SM) mostrou risco quase duplicado quando comparado à categoria de maior renda (> 3,0 SM). O risco de hospitalização para crianças cujas mães apresentavam até quatro anos de escolaridade foi cerca de cinco vezes maior em relação ao grupo de melhor escolaridade ( $\geq 9$ anos), enquanto que para o grupo intermediário o risco foi 2,6 vezes maior. A escolarida-

Tabela 1 - Distribuição dos casos de pneumonia de acordo com características demográficas e socioeconômicas, acompanhada de sua razão de "odds" bruta e nível de significância. Pelotas, 1993.

\begin{tabular}{|c|c|c|c|c|}
\hline Variáveis & $\begin{array}{c}\text { Incidência de } \\
\text { hospitalizações }\end{array}$ & $\begin{array}{c}\text { Número de } \\
\text { crianças }\end{array}$ & $\begin{array}{c}\text { Razão de "O dds" } \\
\text { (IC 95\%) }\end{array}$ & $\begin{array}{c}\text { Nível de } \\
\text { significância }\end{array}$ \\
\hline \multicolumn{5}{|l|}{ Sexo } \\
\hline Masculino & $3,1 \%$ & 2.608 & $1,19(0,86-1,65)$ & 0,29 \\
\hline Feminino & $2,6 \%$ & 2.696 & 1,00 & \\
\hline Classe Social & & & & $<0,001$ \\
\hline Burguesia e nova & & & & $u<0,001$ \\
\hline pequena burguesia & $0,4 \%$ & 260 & $0,20(0,03-1,44)$ & \\
\hline Pequena burguesia tradicional & $2,8 \%$ & 889 & $1,48(0,89-2,46)$ & \\
\hline Proletariado não típico & $1,9 \%$ & 2.037 & 1,00 & \\
\hline Proletariado típico & $3,3 \%$ & 1.260 & $1,77(1,14-2,75)$ & \\
\hline Subproletariado & $6,4 \%$ & 468 & $3,51(2,15-5,71)$ & \\
\hline $\begin{array}{l}\text { Renda familiar em } \\
\text { salários-mínimos mensais } ₫\end{array}$ & & & & $\begin{aligned} & <0,001 \\
u & <0,001\end{aligned}$ \\
\hline$\leq 1$ & $4,7 \%$ & 989 & $2,89(1,83-4,51)$ & \\
\hline 1,1 a 3 & $3,2 \%$ & 2.279 & $1,92(1,27-2,90)$ & \\
\hline$>3$ & $1,7 \%$ & 2.036 & 1,00 & \\
\hline $\begin{array}{l}\text { Anos completos de } \\
\text { escolaridade da mãe }\end{array}$ & & & & $\begin{aligned} & <0,001 \\
u & <0,001\end{aligned}$ \\
\hline Nenhum & $4,4 \%$ & 137 & $4,39(1,66-11,63)$ & \\
\hline 1 a 4 & $4,9 \%$ & 1.362 & $4,95(2,77-8,85)$ & \\
\hline 5 a 8 & $2,7 \%$ & 2.454 & $2,61(1,46-4,68)$ & \\
\hline$\geq 9$ & $1,0 \%$ & 1.352 & 1,00 & \\
\hline $\begin{array}{l}\text { Anos completos de } \\
\text { escolaridade do pai }\end{array}$ & & & & $\begin{aligned} & <0,001 \\
u & <0,001\end{aligned}$ \\
\hline Nenhum & $2,4 \%$ & 124 & $2,07(0,59-7,24)$ & \\
\hline 1 a 4 & $3,9 \%$ & 1.135 & $3,36(1,86-6,07)$ & \\
\hline 5 a 8 & $3,0 \%$ & 2.384 & $2,56(1,46-4,49)$ & \\
\hline$\geq 9$ & $1,2 \%$ & 1.266 & 1,00 & \\
\hline Total & $100 \%$ & 5.304 & & \\
\hline
\end{tabular}

u Teste para tendência linear de proporções (unicaudal)

® Variáveis com informações desconhecidas para mais de $5 \%$ das crianças 
Tabela 2 - Distribuição dos casos de pneumonia de acordo com características reprodutivas maternas e gestacionais acompanhadas da sua razão de "odds" bruta e nível de significância correspondentes. Pelotas, 1993.

\begin{tabular}{|c|c|c|c|c|}
\hline Variáveis & $\begin{array}{l}\text { Percentual de } \\
\text { hospitalizações }\end{array}$ & $\begin{array}{l}\text { Número de } \\
\text { crianças }\end{array}$ & $\begin{array}{l}\text { Razão de "odds" } \\
\text { (IC 95\%) }\end{array}$ & Valor de P \\
\hline $\begin{array}{l}\text { Idade materna em } \\
\text { anos completos }\end{array}$ & & & & $\begin{array}{r}0,49 \\
\text { u } 0,08\end{array}$ \\
\hline$<20$ & $3,8 \%$ & 921 & $1,50(0,93-2,41)$ & \\
\hline 20 a24 & $2,8 \%$ & 1.462 & $1,10(0,63-1,77)$ & \\
\hline 25 a 29 & $2,6 \%$ & 1.365 & 1,00 & \\
\hline 30 a34 & $2,7 \%$ & 962 & $1,06(0,63-1,77)$ & \\
\hline$\geq 35$ & $2,5 \%$ & 592 & $0,99(0,54-1,83)$ & \\
\hline Paridade & & & & $<0,001$ \\
\hline Nenhum & $2,3 \%$ & 1.859 & 1,00 & $u<0,005$ \\
\hline 1 & $2,0 \%$ & 1.470 & $0,90(0,56-1,45)$ & \\
\hline 2 & $2,8 \%$ & 932 & $1,24(0,76-2,04)$ & \\
\hline 3 ou mais & $5,2 \%$ & 1.041 & $2,37(1,57-3,57)$ & \\
\hline $\begin{array}{l}\text { Intervalo interpartal } \\
\text { (em meses)* }\end{array}$ & & & & $\begin{array}{r}0,004 \\
\text { u } 0,29\end{array}$ \\
\hline$<24$ & $4,9 \%$ & 587 & $2,02(1,24-3,30)$ & \\
\hline 24 a 35 & $3,8 \%$ & 526 & $1,54(0,89-2,66)$ & \\
\hline 36 a 47 & $3,8 \%$ & 426 & $1,52(0,84-2,74)$ & \\
\hline$\geq 48$ & $2,5 \%$ & 1.555 & 1,00 & \\
\hline G anho de peso na gestação & & & & 0,003 \\
\hline$<10 \mathrm{~kg}$ & $3,8 \%$ & 1.843 & $1,63(1,18-2,25)$ & \\
\hline$\geq 10 \mathrm{~kg}$ & $2,4 \%$ & 3.467 & 1,00 & \\
\hline $\begin{array}{l}\text { Pré-termo ou retardo de } \\
\text { crescimento intra-uterino (RCIU) }\end{array}$ & & & & 0,16 \\
\hline Pré-termo & $3,7 \%$ & $(437)$ & $1,39(0,82-2,36)$ & \\
\hline $\mathrm{RCIU}$ & $4,1 \%$ & $(441)$ & $1,56(0,94-2,58)$ & \\
\hline O utro & $2,7 \%$ & $(4.432)$ & 1,00 & \\
\hline Total & $100 \%$ & 5.302 & & \\
\hline
\end{tabular}

de do pai mostrou-se menos importante; mesmo assim, o risco de hospitalização ficou entre 2,1 e 3,4 vezes maior em relação à categoria com maior grau de instrução. Tanto para a escolaridade do pai quanto da mãe, o mais alto risco foi observado para a categoria de 1-4 anos.

A Tabela 2 mostra que a idade materna não esteve significativamente associada à hospitalização por pneumonia. Quanto à paridade, crianças cujas mães tiveram três ou mais partos apresentaram risco 2,4 vezes maior em relação às primíparas. Associação inversa foi observada em relação ao intervalo interpartal; quando inferior a 24 meses, o risco mostrou-se duplicado, enquanto que para os grupos intermediários, 24 35 e 36-47 meses, o risco foi cerca de $50 \%$ maior em relação a categoria-base ( $\geq 48$ meses). O risco de hospitalização foi $63 \%$ maior entre filhos de mães com ganho de peso inferior a $10 \mathrm{~kg}$ durante a gestação. Retardo de crescimento intra-uterino e nascimento prétermo estiveram associados com razões de "odds" 1,39 e 1,56, respectivamente, não havendo alcançado significância estatística.
A análise multivariada obedeceu ao modelo hierárquico apresentado na Figura. Todas as variáveis foram testadas através desse processo, sendo eliminadas aquelas que não mostraram associação significativa quando ajustadas, o que ocorreu com escolaridade paterna e intervalo interpartal.

Em um primeiro passo entrou a variável sexo. Embora essa não alcançasse o nível de significância requerido, decidiu-se a priori que seria controlada em todas as análises. Em seguida, incluiu-se a classe social, que manteve a mesma significância observada na análise bruta com razão de "odds" de 3,5 para o subproletariado em relação ao proletariado não típico. No terceiro nível entraram as variáveis renda familiar, escolaridade da mãe e escolaridade do pai, tendo essa última perdido a significância quando ajustada para as demais. A renda familiar perdeu grande parte do seu efeito. Quanto à escolaridade da mãe, manteve-se o risco relativo de 2,7 para o grupo de menor instrução e 3,2 para a categoria de um a quatro anos. No quarto nível foram acrescidas ao modelo as variáveis idade materna e paridade. $\mathrm{O}$ intervalo 
interpartal não foi incluído por deixar de ser significativo após ajuste. Filhos de adolescentes ( $<20$ anos) apresentaram risco duplicado em relação à categoria-base ( 25 a 29 anos). O ganho de peso inferior a $10 \mathrm{~kg}$ na gestação esteve associado com risco $38 \%$ maior de o filho ser hospitalizado no período pósneonatal, mesmo após ajuste para todos os níveis anteriores.

No último nível foram introduzidas, em uma mesma variável, prematuridade e retardo de crescimento intra-uterino que mostraram efeito ainda menor, quando ajustado, sobre a variável dependente.

A realização de consultas de pré-natal, tipo de parto e peso ao nascer não se mostraram significativamente associadas à admissão hospitalar.

\section{ISCU SSÃO}

Ao analisar os resultados da presente pesquisa, duas limitações devem ser levadas em conta. A primeira refere-se ao paradoxo de Berkson ${ }^{1,15}$, um tipo de viés de seleção que confere maior probabilidade de hospitalização a indivíduos que apresentam uma determinada característica. Por exemplo, entre duas crianças com o mesmo quadro clínico, dá-se preferência à hospitalização daquela de pior nível socioeconômico em virtude de sua família não dispor de condições adequadas ao tratamento domiciliar. Isso eleva ainda mais a taxa de hospitalização desse grupo que já apresenta os maiores índices de morbimortalidade ${ }^{19,27,29}$. A segunda limitação diz respeito ao diagnóstico baseado na história clínica e exame físico. Este método, embora bastante sensível, é pouco específico, fato que pode superestimar o verdadeiro número de casos ${ }^{2}$. No entanto, pouquíssimos estudos em todo o mundo tiveram o diagnóstico de pneumonia comprovado radiologicamente, em razão das dificuldades logísticas e do custo eleva$\mathrm{do}^{4,11,30}$. No presente estudo procurou-se contornar esse problema através do processo de arbitragem e da interpretação padronizada das radiografias disponíveis de boa qualidade. $\mathrm{O}$ valor preditivo positivo $(76 \%)$ do diagnóstico clínico, em relação ao radiológico, leva a crer que esta limitação pouco afetou os resultados. Por fim, a ocorrência de hospitalizações em outras cidades parece pouco provável em virtude de Pelotas ser o segundo maior centro médico do Rio Grande do Sul. São duas Faculdades de Medicina, cerca de 1.200 médicos e 400 enfermeiros, cinco hospitais, quatro unidades de terapia pediátrica entre outros. Embora seja possível que algumas crianças possam ter sido hospitalizadas na capital (distante cerca de $250 \mathrm{~km}$ ), é de se supor, pelo acima exposto, que isto tenha ocorrido muito raramente. Em vista disso, do ponto de vista estatístico, não afetariam os resultados encontrados. Além disso, monitorizar a hospitalização dessas crianças em dezenas de hospitais em Porto Alegre seria logisticamente impraticável, além de elevar substancialmente o custo do estudo.

Diversos estudos demonstram que a ocorrência de pneumonia é maior entre meninos ${ }^{5,18,21,32}$, com inversão de risco a partir dos seis meses de idade ${ }^{11,30}$. No presente estudo, embora os meninos tenham sido hospitalizados em maior freqüência, as diferenças observadas não alcançaram significância estatística.

A morbimortalidade infantil é substancialmente maior entre crianças de classe social baixa ou de menor poder aquisitivo ${ }^{5,11,21,27,30,32}$. Estudo realizado na década de 80 em Pelotas mostrou que a taxa de hospitalização por infecções respiratórias agudas, até os 20 meses de idade, foi cinco vezes maior para crianças com renda familiar de até $1 \mathrm{SM}$ em relação àquelas com renda mensal superior a $6 \mathrm{SM}^{3}$. Os dados obtidos no presente estudo mostram que a classe social foi o mais importante determinante das hospitalizações em virtude dos riscos observados - cerca de 20 vezes menor para a categoria de menor risco (burguesia) em relação a de maior risco (subproletariado). O efeito isolado da renda praticamente desapareceu após ajuste para a classe social por causa da alta associação entre estas duas variáveis.

A baixa escolaridade materna está associada a um risco aumentado de hospitalização e de mortalidade por pneumonia nos primeiros anos de vida ${ }^{28,30,32}$. Estudo realizado em Porto Alegre ${ }^{30}$ mostrou que a hospitalização foi $40 \%$ menor entre crianças cujas mães possuiam oito anos ou mais de escolaridade quando comparada aquelas com dois anos ou menos. Os riscos relativos obtidos, após ajustes, mostram que a escolaridade materna apresenta papel independente na etiologia das hospitalizações por pneumonia.

Filhos de mães adolescentes ( $<20$ anos), ou com idade igual ou superior a 35 anos, adoecem e morrem por pneumonia com maior freqüência em virtude da menor experiência na identificação e manejo dos casos graves ${ }^{19,27,30}$. O risco relativo à hospitalização para filhos de adolescentes foi duas vezes superior à categoria de menor risco. Este achado deve ser levado em conta na identificação de crianças de risco.

Assim, como em outros estudos ${ }^{19,26,30}$, a paridade esteve diretamente associada à hospitalização por pneumonia no período pós-neonatal. Esta tendência pode ser claramente observada no presente estudo. $\mathrm{O}$ risco relativo mostrou-se triplicado para crianças cujas mães tiveram três ou mais partos. Tendência 
Tabela 3 - Modelo de regressão logística hierárquica múltipla para fatores de risco socioeconômicos e reprodutivos maternos para pneumonia pós-neonatal com a razão de odds e nível de significância correspondente. Pelotas, 1993.

\begin{tabular}{|c|c|c|}
\hline Variáveis & Razão de “O dds" (IC 95\%) & Nível de significância \\
\hline Sexo $^{1}$ & & \\
\hline M asculino & $1,16(0,84-1,60)$ & 0,36 \\
\hline Feminino & 1,00 & \\
\hline Classe Social $^{2}$ & & $u<0,001$ \\
\hline $\begin{array}{l}\text { Burguesia e nova pequena } \\
\text { burguesia }\end{array}$ & $0,20(0,27-1,44)$ & \\
\hline Pequena burguesia tradicional & $1,49(0,89-2,47)$ & \\
\hline Proletariado não típico & 1,00 & \\
\hline Proletariado típico & $1,78(1,14-2,76)$ & \\
\hline Subproletariado & $3,50(2,15-5,70)$ & \\
\hline Renda familiar em salários-mínimos mensais ${ }^{3 a}$ & & u 0,10 \\
\hline$\leq 1$ & $1,47(0,89-2,43)$ & \\
\hline 1,1 a 3 & $1,30(0,84-2,01)$ & \\
\hline$>3$ & 1,00 & \\
\hline Anos completos de escolaridade da mãe ${ }^{3 a}$ & & $u<0,001$ \\
\hline Nenhum & $2,70(0,98-7,45)$ & \\
\hline 1 a 4 & $3,24(1,73-6,09)$ & \\
\hline 5 a 8 & $1,97(1,07-3,61)$ & \\
\hline$\geq 9$ & 1,0 & \\
\hline Anos completos de escolaridade do pai ${ }^{3 b}$ & & $u<0,5$ \\
\hline Nenhum & $0,69(0,19-2,57)$ & \\
\hline 1 a 4 & $1,38(0,71-2,70)$ & \\
\hline 5 a 8 & $1,37(0,74-2,53)$ & \\
\hline$\geq 9$ & 1,00 & \\
\hline Idade materna em anos completos & & u 0,08 \\
\hline$<20$ & $1,98(1,12-3,51)$ & \\
\hline 20 a24 & $1,32(0,82-2,13)$ & \\
\hline 25 a 29 & 1,00 & \\
\hline 30 a 34 & $0,96(0,56-1,63)$ & \\
\hline$\geq 35$ & $0,75(0,40-1,42)$ & \\
\hline Paridade $^{4 a}$ & & $\mathrm{u}<0,01$ \\
\hline Nenhum & 1,00 & \\
\hline 1 & $1,05(0,64-1,74)$ & \\
\hline 2 & $1,53(0,87-2,68)$ & \\
\hline 3 ou mais & $2,86(1,64-4,99)$ & \\
\hline Intervalo interpartal (em meses) ${ }^{4 b}$ & & u 0,55 \\
\hline$<24$ & $1,34(0,79-2,28)$ & \\
\hline 24 a 35 & $1,22(0,66-2,24)$ & \\
\hline 36 a 47 & $1,12(0,63-2,00)$ & \\
\hline$\geq 48$ & 1,00 & \\
\hline Ganho de peso ${ }^{5}$ & & 0,05 \\
\hline$<10 \mathrm{~kg}$ & 1,00 & \\
\hline$\geq 10 \mathrm{~kg}$ & $1,38(1,00-1,92)$ & \\
\hline Pré-termo ou retardo de crescimento intra-uterino 6 & & 0,46 \\
\hline Pré-termo & $1,19(0,69-2,05)$ & \\
\hline $\mathrm{RCIU}$ & $1,33(0,80-2,23)$ & \\
\hline O utro & 1,00 & \\
\hline
\end{tabular}

u Teste para tendência linear de proporções (unicaudal)

$2-1+$ classe social

a - 2 + escolaridade da mãe + renda familiar

b - 2 + escolaridade da mãe + renda familiar + escolaridade do pai

$4 a$ - 3a + idade da mãe + paridade

$4 b$ - $3 a+$ idade da mãe + paridade + intervalo interpartal

5 - $4 a+$ ganho de peso durante gestação 
semelhante foi encontrada para o intervalo interpartal, porém, não significativa do ponto de vista estatístico. Chama a atenção o fato de o intervalo interpartal ser proximal ao desfecho (Figura) e de não exercer influência importante sobre as hospitalizações. Em três outros estudos realizados recentemente no Brasil, essa variável perdeu a significância estatística quando ajustada para os fatores de confusão $0^{11,27,30}$.

O ganho de peso durante a gestação tem sido descrito como o principal determinante do peso ao nas$\operatorname{cer}^{5,16,26,32}$. No entanto, não se encontrou na literatura revisada referência sobre a associação entre o ganho de peso neste período e a ocorrência de hospitalização por pneumonia no primeiro ano de vida. Os filhos cujas mães ganharam menos de 10 quilos durante a gestação apresentaram risco cerca de $40 \%$ maior à hospitalização por pneumonia em relação às demais crianças. Vale lembrar que cerca de um terço das mães não foram pesadas no início da gestação, sendo anotado o peso referido imediatamente antes da gravidez, o qual pode ter sido afetado por erros de recordatório. Mesmo assim, não há razão para crer que as mães cujos filhos fossem hospitalizados por pneumonia recordassem o peso de forma diferente das demais. Por outro lado, em contraste com outros estudos $^{11,19,26,30}$, o baixo peso ao nascer e a prematuridade não estiveram significativamente associados à admissão hospitalar por pneumonia. Desta forma, parece que o efeito do ganho de peso materno não é mediado pelo crescimento fetal. Há necessidade de pesquisas que confirmem este achado. Embora não se possa descartar a possibilidade de uma associação fortuita, seria interessante explorar outros meca-

\section{REFERÊN CIAS BIBLIO GRÁFICAS}

1. BERKSON, J. Limitations of the aplications of four-fold table analysis to hospital data. Biom. Bull., 2:47-53, 1946.

2. BERMAN, S. Overwiew of pneumonia in early infancy. In: Acute Lower Respiratory Infection \& Child Survival in developing countries. Baltimore, The Johns Hopkins University, 1990. p 12-27.

3. BRONFMAN, M.; LOMBARDI, C.; FACCHINI, L. A.; VICTORA, C.G.; BARROS, F.C. Operacionalização do conceito de classe social em estudos epidemiológicos. Rev. Saúde Pública, 22:253-65, 1988.

4. CHANDRA, R. K. Prospective studies of the effect of breast feeding on the incidence of infection and allergy. Acta Paediatr. Scand., 68:691-4, 1979.

5. CHILDHOOD pneumonia: strategies to meet the challenge. In: Proceedings of the International Consultation on Acute Respiratory Infections, Washington, D.C.,1991. London, Appropriate Health Resources and Technology Action Group - AHRTAG, 1992. nismos que pudessem explicar esta associação, como, por exemplo, um desenvolvimento mais rápido do sistema imunológico naquelas crianças cujas mães apresentam melhor estado nutricional.

Estudar morbidade é uma tarefa bastante difícil visto que nem todos os doentes procuram o serviço de saúde ou, quando o fazem, como no caso das hospitalizações, outros critérios que não somente o quadro clínico do paciente determinam a prestação de cuidados. Mesmo assim, o presente estudo mostrou alguns avanços em relação aos demais. Do ponto de vista metodológico: i) possibilitou o acompanhamento de uma coorte representativa de crianças em uma cidade de porte médio, evitando o viés de seleção; ii) revelou baixos índices de perdas através da revisão concomitante dos prontuários e imediata entrevista com o médico, limitação constante nos estudos que tratam deste assunto; iii) determinou o alto valor preditivo positivo da avaliação clínica da causa de internação em relação ao diagnóstico radiológico.

No que se refere aos resultados, o estudo reforçou a importância dos fatores socioeconômicos (classe social, escolaridade materna), cuidados maternos (escolaridade e idade), intensidade de exposição (paridade) e condição nutricional (ganho de peso durante a gestação) sobre a hospitalização por pneumonia no período pós-neonatal. Esses achados podem contribuir para a melhoria dos serviços oferecidos à população, quer seja por proporcionar melhor entendimento de um evento bastante freqüente no País, como também por permitir a identificação precoce de crianças com risco aumentado de adoecer e morrer por pneumonia neste período.

6. CONFERÊNCIA MUNICIPAL DE SAÚDE DE PELOTAS. Pelotas, Secretaria Municipal de Saúde e Meio Ambiente, 1994.

7. DEAN, A. G.; DEAN, J. A.; COULOMBIER, D.; BRENDEL, K. A.; SMITH, D.C.; BURTON, A. H.; DICKER, R. C.; SULLIVEN, K.; FAGAN, R.F.; ARNER, T. G. Epi Info, version 6: a word processing, database, and statistics program for epidemiology on microcomputers. Atlanta,Centers of Disease Control and Prevention,1994.

8. DUBOWITZ, L. M. S.; DUBOWITZ, V.; GOLDBERG, C. Clinical assesment of gestacional age in the newborn infant. J. Paediatr., 77: 1-10, 1970.

9. EPIDEMIOLOGICAL graphics, estimation and testing package - EGRET. Washington, D. C., Statistics and Research Corporation, 1988.

10. ESTAÇÃO AGROCLIMATOLÓGICA EMBRAPAUFPEL. Dados normativos: últimos 30 anos. Bol. Meteor., jul., 1995. 
11. FONSECA, W.; KIRKWOOD, B. R.; VICTORA, C. G.; FUCHS, S. R.; FLORES, J. A.; MISAGO, C. Risk factors for childhood pneumonia among the urban poor in Fortaleza Brazil: a case-control study. Bull. World Health Organ., 74: 199-208, 1996.

12. FUNDAÇÃO IBGE / UNICEF. Indicadores sociais: crianças e adolescentes. Rio de Janeiro, 1994. p.19,40 [Censo Demográfico, Rio Grande do Sul -1991. Edição especial].

13. HARARI, H.; SHANN, F.; SPOONER, V.; MEISNER, S.; CARNEY, M.; CAMPO, J. Clinical signs of pneumonia in children. Lancet, 338: 928-30, 1991.

14. JAMISON, D.T.\& MOSLEY, H. Disease control priorities in developing countries. New York, Oxford University Press for the World Bank, 1991.

15. LILIENFELD, D.L. \& STOLEY, P. D. Foundations of epidemiology: case -control studies. 3rd. ed. London, Oxford University Press, 1994.

16. MILLER, J. E. \& ELLERTSON C. E. Intervention to reduce morbidity and mortality from pneumonia in children: encouraging birth spacing; rewiew of potential intervention to reduce morbidity and mortality from pneumonia in children; child spacing; draft. Geneva, LSTHM/WHO, 1993. p. 1-43.

17. ORGANIZAÇÃO MUNDIAL DA SAÚDE. Classificação Internacional de Doenças; $9^{\mathrm{a}}$ rev. 1975. Porto Alegre, Editora Sagr-DC Luzzatto, 1979.

18. PARKER, L. R. Acute respiratory illness in children: PHC responses. Health Policy Plann., 2:279-88, 1987.

19. PIO, A.; LEOWSKI, J.; DAM, H. G. The magnitude of the problem of acute respiratory infections. In: Douglas, R. M. $\&$ Kerby-Eaton, E. Acute respiratory infections in childhood. Adelaide, University of Adelaide, 1985. p. 100-3 [Proceedings of an International Workshop, Sydney, 1984].

20. PUFFER, R. R. \& SERRANO, C. V. Patterns of mortality in childhood: report of the inter-American investigation of mortality in childhood. Washington, D.C., Panamerican Health Organization,1973. (PAHO-Scientific Publications, 263).

21. SCHWARTZ, B.; LIPMAN, H.; LOB-LEVYT, J.; GOVE, S. The etiology of acute lower respiratory infections among young children in developing countries, Geneva, World Health Organization, 1994.

22. SECRETARIA DO DESENVOLVIMENTO ECONÔMICO E SOCIAL. Aspectos socioeconômicos dos municípios do Rio Grande do Sul - 1991-92. Porto Alegre, 1993.
23. SELWYN, B. J. The epidemiological of acute respiratory tract infection in young children: comparison of findings from several developing countries. Rev. Infec. Dis., 12:S870-88, 1990.

24. SHANN, F. A. Respiratory infections in children: management at small hospitals; Background notes and a manual for doctors. Geneva, Wolrd Health Organization, 1986. (9WHO/ RSD/86.26 rev.1).

25. SMITH, P.G. \& DAY, N. E. The design of case-control studies: the influence of confounding and interactions effects. Int. J. Epidemiol., 13: 356-65, 1984.

26. VICTORA, C.G.; SMITH, P. G.; VAUGHAN, J. P.; BARROS, F. C.; FUCHS, S. C.; GIGANTE, L.; NOBRE, L. Influence of birtweight on infectious diseases mortality: a case-control study. Pediatrics, 81:807-11, 1988.

27. VICTORA, C. G.; SMITH, P.G.; BARROS, F. C. VAUGHAN, J. P.; FUCHS, S. C. Risk factors for deaths due to respiratory infections among Brazilian infants. Int. J. Epidemiol., 18:918-25,1989.

28. VICTORA, C. G.; BARROS, F.C.; KIRKWOOD, B. R.; VAUGHAN, J. P. Pneumonia, diarrhoea and growth in the first four years of life: a longitudinal study of 5,914 Brazilian Infants. Amer. J. Clin. Nutr., 52:391-6, 1990.

29. VICTORA, C. G.; BARROS, F.C.; VAUGHAN, J. P Hospitalização e doenças. In: Epidemiologia da desigualdade: um estudo de 6000 crianças brasileiras. $2^{\mathrm{a}}$ ed., São Paulo, Hucitec, 1991. p. 86-93.

30. VICTORA, C. G.; FUCHS, S. C.; FLORES, J. A.; FONSECA, W.; KIRKWOOD, B. Risk factors for pneumonia among children in a Brazilian metropolitan. Pediatrics, 93:977-85,1994.

31. VICTORA, C. G.; BARROS, F. C.; HALPERN, R.; MENEZES, A. M. B.; HORTA, B. L.; TOMASI, E.; WEIDERPASS, E.; CÉSAR, J. A.; OLINTO, M. T. A.; GARCIA, M. M.; VAUGHAN, J. P. Estudo longitudinal da população materno-infantil da região urbana do sul do Brasil,1993: aspectos metodológicos e resultados preliminares. Rev. Saúde Pública,30 34-45,1995.

32. VICTORA, C. G. Risk factors for lower acute respiratory infections. In: Benguigui, Y.; Lopez Antuñano, F. J.; Yunes, J. Infecciones respiratórias agudas en niños. Washington, D. C., Organización Panamericana de la Salud,1995. p.1-38 (no prelo).

33. WILLIANS, R. L.; CREASY, R. K.; CUNNINGHAM, G. C.; HAWENS, W. E.; NORRIS, F. D.; TASHIRO, M. Fetal growths and perinatal viability in California. Obstet. Gynecol., 59:624-32, 1982. 